\title{
Efficient robotic path planning algorithm based on artificial potential field
}

\author{
Elia Nadira Sabudin', Rosli Omar ${ }^{2}$, Sanjoy Kumar Debnath ${ }^{3}$, Muhammad Suhaimi Sulong ${ }^{4}$ \\ ${ }^{1,2,3}$ Faculty of Electrical and Electronic Engineering, Universiti Tun Hussein Onn Malaysia, Johor, Malaysia \\ ${ }^{4}$ Faculty of Technical and Vocational Education, Universiti Tun Hussein Onn Malaysia, Johor, Malaysia
}

\section{Article Info}

\section{Article history:}

Received Sep 1, 2020

Revised Feb 28, 2021

Accepted Mar 3, 2021

\section{Keywords:}

Artificial potential field

Path planning

Visibility graph

\begin{abstract}
Path planning is crucial for a robot to be able to reach a target point safely to accomplish a given mission. In path planning, three essential criteria have to be considered namely path length, computational complexity and completeness. Among established path planning methods are voronoi diagram (VD), cell decomposition (CD), probability roadmap (PRM), visibility graph (VG) and potential field (PF). The above-mentioned methods could not fulfill all three criteria simultaneously which limits their application in optimal and real-time path planning. This paper proposes a path PF-based planning algorithm called dynamic artificial PF (DAPF). The proposed algorithm is capable of eliminating the local minima that frequently occurs in the conventional PF while fulfilling the criterion of path planning. DAPF also integrates path pruning to shorten the planned path. In order to evaluate its performance, DAPF has been simulated and compared with VG in terms of path length and computational complexity. It is found that DAPF is consistent in generating paths with low computation time in obstacle-rich environments compared to VG. The paths produced also are nearly optimal with respect to VG.
\end{abstract}

This is an open access article under the CC BY-SA license.

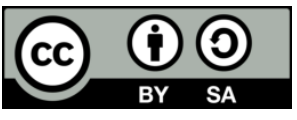

\section{Corresponding Author:}

Rosli Omar

Faculty of Electrical and Electronic Engineering

Universiti Tun Hussein Onn Malaysia

86400 Parit Raja, Batu Pahat, Johor, Malaysia

Email: roslio@uthm.edu.my

\section{INTRODUCTION}

Path planning is among the important aspects that need to be considered in enhancing robot autonomy level [1], [2]. In robotics, path planning is the task of finding a collision-free and feasible path for a robot to traverse from an initial point to a target point. The path planning problem concentrates on three criteria; computational time, optimal path and completeness [3]. Computation time relates exponentially to the number of degrees of freedom of the robot and is affected by the number of obstacles in the environment. An optimal path, which is referred to a shortest distance from the starting point to the target point, is essential to minimize the energy consumption and time needed for the robot to accomplish its mission. Finally, completeness means that the robot can to produce a path if one exists [4]. Once a robot is required to move from a starting point to a target point, the robot needs to produce the shortest path to reach the target safely with low computational time. In a real-time applications, low computation time is vital.

There are several popular methods used for path planning such as voronoi diagram (VD), cell decomposition (CD), visibility graph (VG), and artificial potential field (APF) to name a few [5]-[13]. CD is commonly used in outdoor scenarios. This method introduces the configuration space $(\mathrm{C}$-Space) by a simple 
and connected region named cells. These cells are discrete and do not overlap with each other in the region, but each cell is adjacent to one another. A connectivity graph is then developed and a graph search algorithm is consequently used to generate a path. A larger cell size makes the path sub-optimal, whereas a smaller size will increase its computational time [14].

VD defines nodes that are equidistant from all the nearest obstacles in C-Space. This method is capable in generating a safe path with a low risk of local minima due to the edges of the path being located quite far from the obstacles. However, VD is incompetent to yield an optimal path [5]. VG is capable of producing an optimal path without possibilities of local minima, which is easy to implement. The generated path is optimal, but its computation time increases as the number of obstacles in C-Space increases [15]-[17].

As for PF, it is simple, fast and highly safe [18]-[20]. PF generates two kinds of forces. The attractive force created by the target point pulls the robot to it; whilst the obstacles generate a repulsive force that keeps the robot from it. The robot direction was determined by the total force. PF is ideal for real-time environments since this method produces a single path with low computation time [21]. However, PF has a significant drawback known as local minima that causes the robot unable in producing a path.

Due to the above-mentioned advantages of PF, this paper proposes a PF-based path planning algorithm called dynamic artificial potential field (DAPF) that successfully eliminates the local minima problem and is able to generate a sub-optimal and collision-free path in obstacle-rich environments with low computation time. In order to further improve the planned path optimality, the path pruning technique is applied. A simulation has been conducted to evaluate the capabilities of DAPF and VG in various scenarios in terms of computation time and path length.

\section{OVERVIEW OF POTENTIAL FIELD}

Artificial potential field (APF) is one of the most common path planning techniques. It is based on the idea of two forms of forces called attractive force and repulsive force. Many researchers have utilized this method due to its characteristics such as elegant, safe and simple [22]. APF was first proposed by Khatib in [23] where the robot is seen as a point influenced by fields created by the starting point, target point and obstacles within the search space. The target point generates an attractive force, while the starting point and obstacles generate a repulsive force.

APF technique has benefits like real-time path planning application due to its; i) low computational time, and ii) capability to produce a collision-free path. On the other hand, the technique suffered from its main disadvantages including local minima, goal not-reachable problem, and narrow passages [24], [25]. A number of researchers have implemented the APF technique for path planning. Li et al. proposed a PF and regression search path planning method for the robot. It was claimed to effectively generate a global suboptimal/optimal path and minimize local minima and oscillation problems in a known environment with incomplete information [26]. Sfeir et al. Performed real-time mobile robot navigation in an unknown environment based on improved APF technique to develop a steady trajectory around the obstacles by evolving integration of rotational force [27]. The present technique efficaciously avoided the hindrance in APF due to goal non-reachable when obstacles are nearby (GNRON) problem. Research by Park et al. presented a potential field method and vector field histogram (VFH) to overtake the PF barriers by creating a new obstacle avoidance method for mobile robots based on advanced fuzzy PFM (AFPFM) [28].

Lifen et al. enhanced the APF by replacing the repulsive potential function that assists an unmanned aerial vehicle (UAV) in generating an optimal path while avoiding collision with obstacles [20]. Li et al. proposed an improved APF in robot path planning, which reconsidered the potential function to compute a legitimate path and therefore minimalize the length of the generated path [29]. Debnath et al. have described the APF as practical to produce a terser path [30].

\section{POTENTIAL FIELDS METHOD FOR PATH PLANNING}

\subsection{Field function based on traditional $\mathbf{P F}$}

The potential field method produces two kinds of force; the attractive force and the repulsive force. The target point generates an attractive force by pulling the robot close to it. In the meantime, the repulsive force is generated by the obstacle and the starting point to repel the robot away from it. The resultant total force will generate the path for the robot. The attractive potential field, $V_{g}$ at target point is defined as (1).

$$
V_{g}=K_{g} r_{g}
$$

Where $K_{g}$ is the attractive gain that is is equal to or greater than zero and $r_{g}$ is the distance between the present robot position and the goal. 
The repulsive potential field, $V_{o}$ produced at obstacle can be represented as (2).

$$
V_{o}=\frac{K_{o}}{r_{o}}
$$

Where $K_{o}$ is the repulsive gain at obstacle which is larger than zero and $r_{o}$ is the distance from the robot. The repulsive potential field, $V_{r}$ generated by the starting point can be written as (3).

$$
V_{r}=\frac{K_{r}}{r_{r}}
$$

Where $K_{r}$ is the repulsive gain at starting point that is equal to or greater than zero and $r_{r}$ is the distance between the present robot position and the starting point. Thus, the total potential field can be written as in (4). The potential fields are illustrated in Figure 1(a)-(c) [13].

$$
V_{\text {total }}=V_{g}+V_{r}+V_{o}
$$

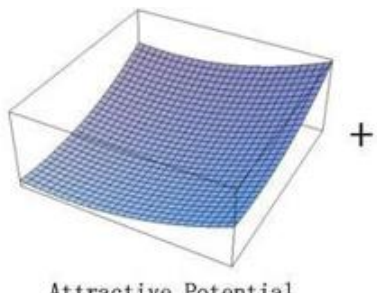

Attractive Potential

(a)

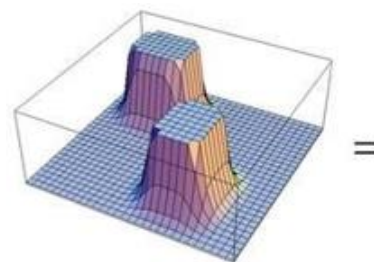

Repulsive Potential

(b)

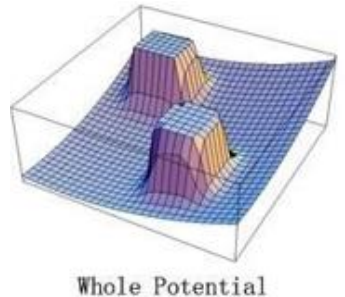

(c)

Figure 1. These figures are; (a) the attractive potential generated by the target point; (b) the repulsive potential generated by the obstacle and starting point; (c) the resulting potential fields

\subsection{The proposed algorithm}

\subsubsection{Parameters setting}

In this paper, we propose a path planning algorithm based on PF called dynamic artificial PF (DAPF). In DAPF, there are some important parameters namely sampling distance $d h$ and the number of discrete points $p$ along the perimeter of an obstacle that will determine the computation time of the algorithm. These parameters have to be set adequately in order to minimize the computation time while guaranteeing the path is collision-free. As such, the parameter values are dynamic depending on the scenario in which the path planning takes place. Through simulations, $d h$ and $p$ can be found from the (5), (6).

$$
\begin{aligned}
& d h=\frac{0.085 \times O}{3500} \\
& p=(p \times 4)-4
\end{aligned}
$$

$O$ in (5) represents the obstacle number in a scenario. In PF, a two-dimensional mesh in $x$ and $y$ axes that covers the entire search space has to be created first. The mesh consists of smaller grid lines whose number is determined by $d h$ and the range of the search space $X Y$. The smaller the value of $d h$, the higher the number of grid lines, which will increase the computation time. Hence a suitable $d h$ value has to be set adequately. By making the mesh size dynamics, the computation time could be improved, especially in a non-obstacle-rich environment to generate a free-collision path. As for the attractive gain at the target point,

$$
K_{g}=\frac{0.8}{\sqrt{(X)^{2}+(Y)^{2}}}
$$

where $X$ and $Y$ represent the ranges of the search along $x$ and $y$ axes, respectively. Contrarily, the repulsive gain at the obstacle, $K_{o}$ and the repulsive gain at the starting point, $K_{r}$ are stated as (8), (9).

$$
K_{o}=\frac{\sqrt{(X)^{2}+(Y)^{2}}}{(p \times o)}
$$




$$
K_{r}=10 \times d h
$$

$K_{o}$ is determined referred on the surrounding dimension (search space diagonal distance) and the sum of obstacles, meanwhile $K_{r}$ is a function of $d h$.

\subsubsection{The algorithm}

In DAPF, the potential field $V_{\text {total }}$ is first generated using equations derived in Sections 3.1. Next, the initial point $x_{i}$ is set to the starting point $x_{S}$. Consequently, the robot starts to maneuver from $x_{i}$ to the subsequent point $x_{l}$ by identifying and selecting the lowest point out of eight surrounding points produced by the potential field. It will then move to $x_{l}$ once the lowest point has been selected. If the point is a local minima, the next lowest point will be identified. This process is repeated until $x_{l}$ is no longer a local minima and $x_{i}$ is then updated to $x_{l}$. The process repeats until $x_{i}=x_{T}$ (target point). The pseudocode of the DAPF algorithm is presented in Figure 2 and the flowchart of the DAPF algorithm is shown in Figure 3. The proposed DAPF is capable of eliminating the local minima problem, oscillation, goal non-reachable problem, and narrow passages. Besides that, it can also produce a path with low computation time so that the robot can be deployed in a real-time mission.

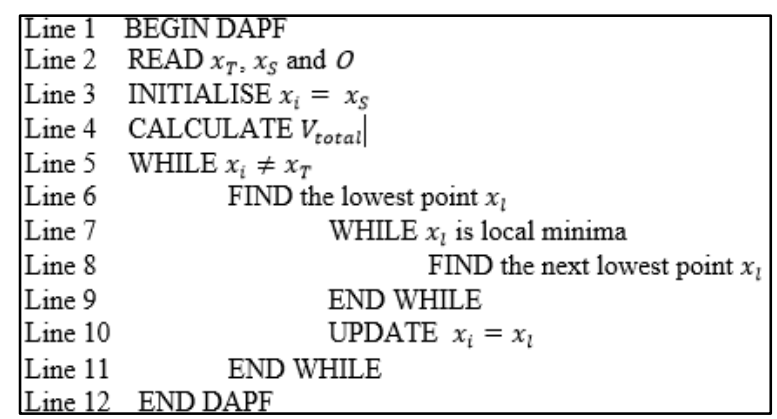

Figure 2. DAPF algorithm

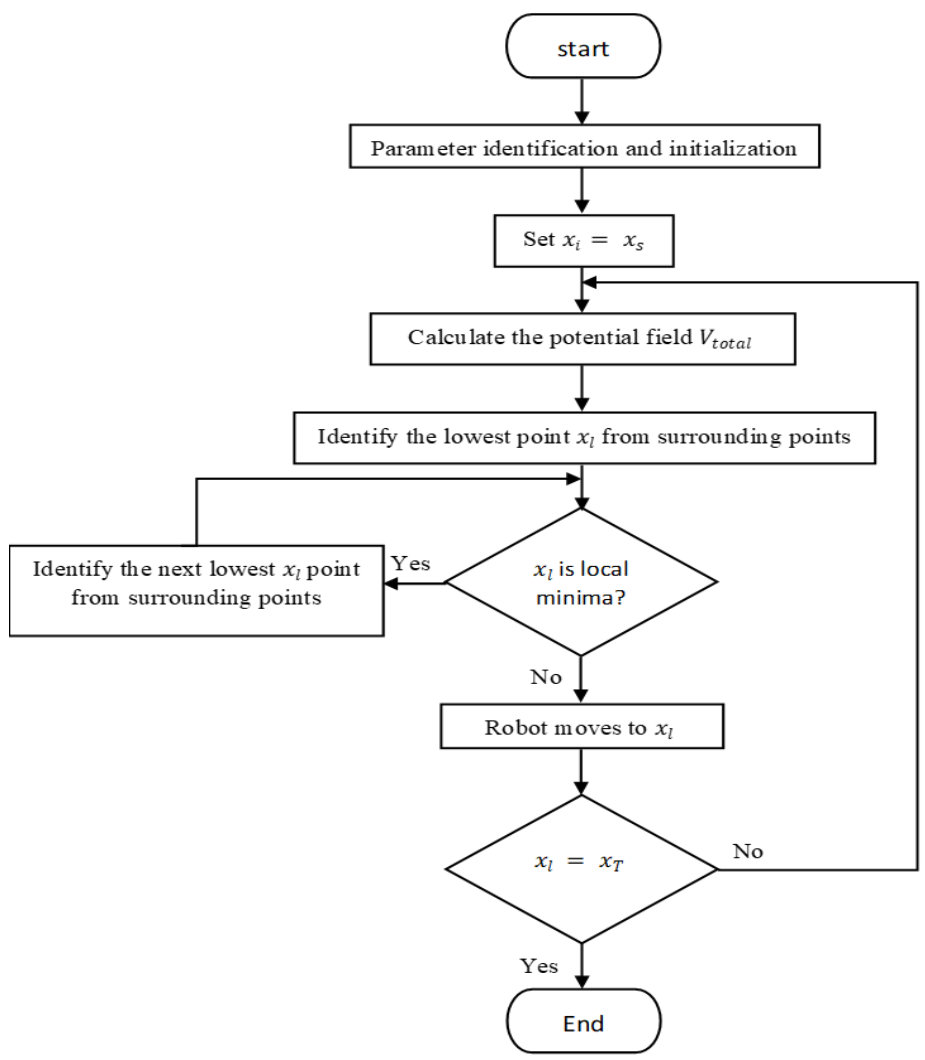

Figure 3. The flowchart of DAPF 


\subsection{Path pruning}

After a path has been generated by DAPF, the path can normally be shortened by passing the areas with high potential values avoiding all the obstacles. Therefore, a path pruning method is applied and its algorithm is shown in Figures 4 and 5. Path pruning makes the robot to be able to perform the mission with a shorter path and hence saves its energy.

A path is a series of waypoints that consists of $\left\{p_{i}, p_{i+1}, p_{i+2}, \ldots p_{n}\right\}$ where $p_{i}$ is the starting point and $p_{n}$ is the target point. The pruning procedure begins with scanning the obstacles between $p_{i}$ and $p_{i+\mathrm{j}}$. If there is no obstacle between $p_{i}$ and $\mathrm{p}_{i+\mathrm{j}}$, the algorithm will eliminate $p_{i+\mathrm{j}-1}$ from $W$ and the value $j$ is updated to $j+1$. On the other hand, if there are obstacles between $p_{i}$ and $p_{i+\mathrm{j}}, p_{i}$ is then updated to $p_{i+\mathrm{j}-1}$ and $j=j+1$, and the algorithm continues to scan obstacles between the updated $p_{i}$ and $p_{i+\mathrm{j}}$. The process ends if $p_{i}=p_{n}$.

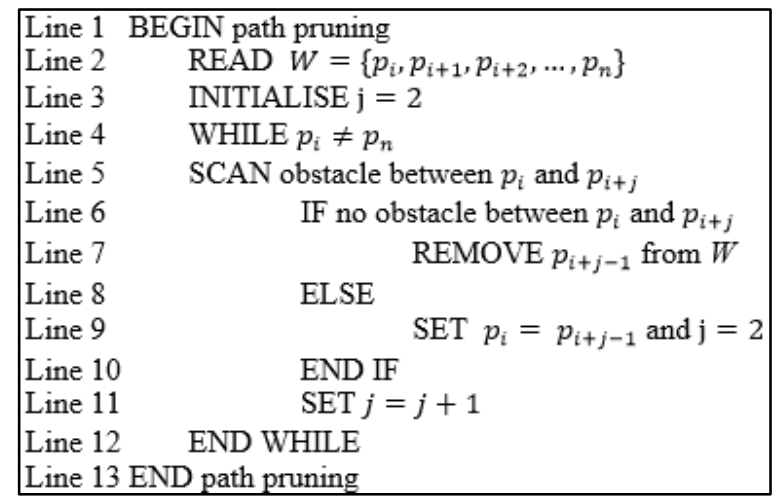

Figure 4. The pseudocode for path pruning

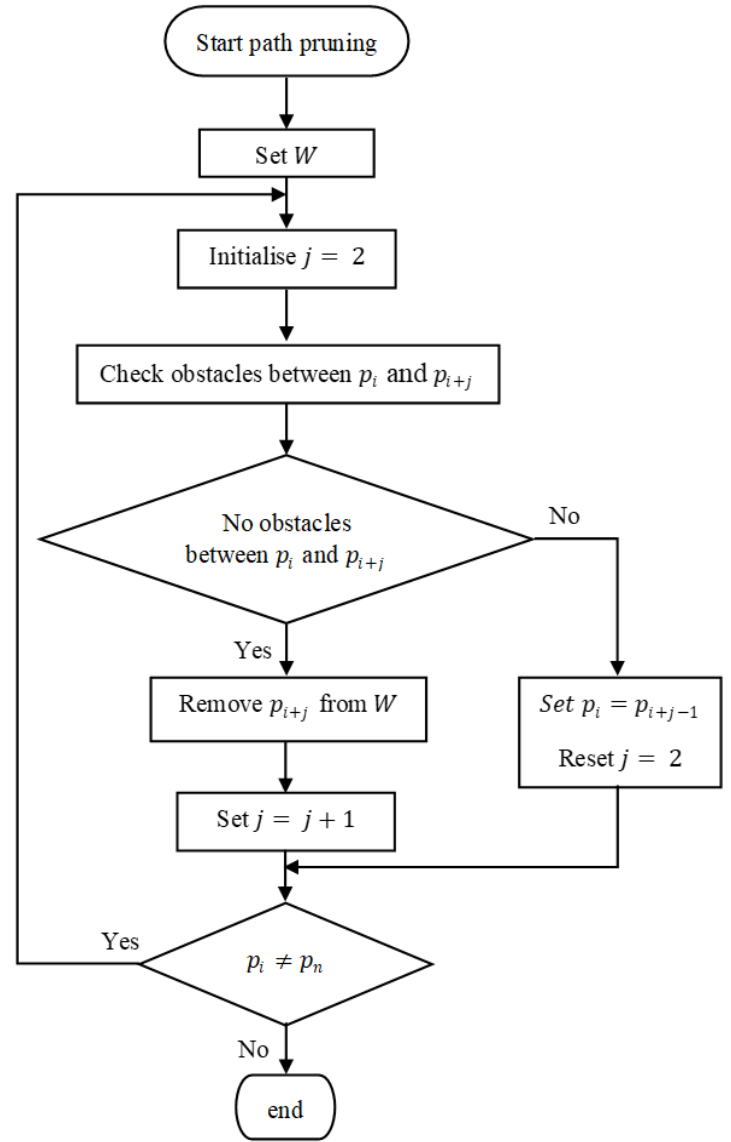

Figure 5. Flowchart for path pruning 


\section{SIMULATION RESULTS AND DISCUSSION}

\subsection{Computation time of DAPF and VG}

A simulation has been performed to assess the performance of the proposed DAPF from the aspect of computation time and path length in random scenarios with a variety number of obstacles. Besides that, a simulation of VG has also been done in identical scenarios to compare its performance against DAPF. The simulation of both methods has been executed in 100 random scenarios with varied obstacles number between 10 to 200. It has successfully been implemented using MATLAB R2016a on a PC with Intel i54200U 1.6 GHz CPU and Windows 10 OS. The ranges of $X$ and $Y$ were set to 750 units. Figure 6 shows the simulation in terms of computation time for DAPF and VG methods. It also shows the result of minimum, average and maximum computation time against the number of obstacles for the DAPF and VG methods.

From Figure 6, it can be observed that the computation time of both DAPF and VG methods were exponentially increased but at a different pace. According to Figure 6(a), with 70 obstacles (green line) in the scenario, it shows that the computation time increases due to local minima. The algorithm took time to eliminate the problems and replanned a new path. Figure 6(b) shows a fluctuated computation times but it seems intangible. VG method resulted in low computation time at $5 \mathrm{~s}$ for a number of obstacles below 80 . Between 100 and 200 obstacles, the computation time of VG was between $7 \mathrm{~s}$ to $32 \mathrm{~s}$. Meanwhile, the computation time for the DAPF method for all scenarios was below $7 \mathrm{~s}$.

Table 1 shows the result of minimum, average and maximum computation time of DAPF and VG in environments with 50, 100, 150 and 200 obstacles, each generated in 100 random scenarios. The computation time of the two methods in finding a collision-free path increased with the increment of the number of obstacles. From Table 1, DAPF produced relatively shorter computation times in all scenarios. The computation times for DAPF were slightly increased in scenarios with 50 and 200 obstacles with average of 0.34 and 6.15 seconds, respectively. However, the increment of computation time of VG in environments with 50 and 200 obstacles was abrupt with an average of 1.58 and 31.23 seconds, respectively. Note that, throughout the simulation, there was no single occasion that the algorithm failed to plan a path. It proves that the algorithm is complete, i.e., manages to find a path if one exists.

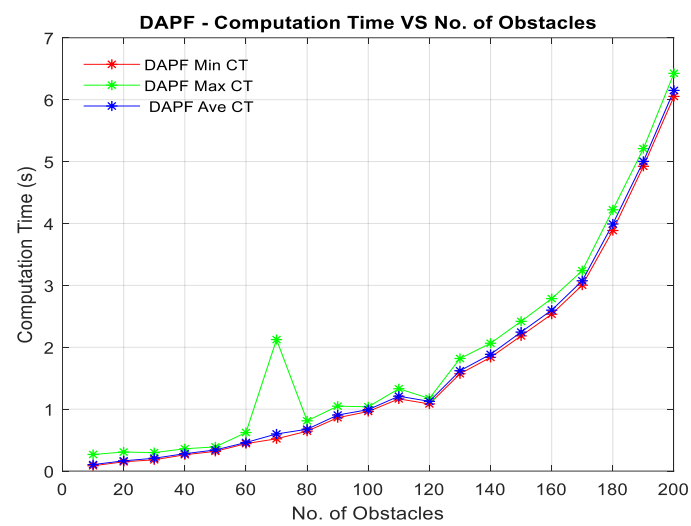

(a)

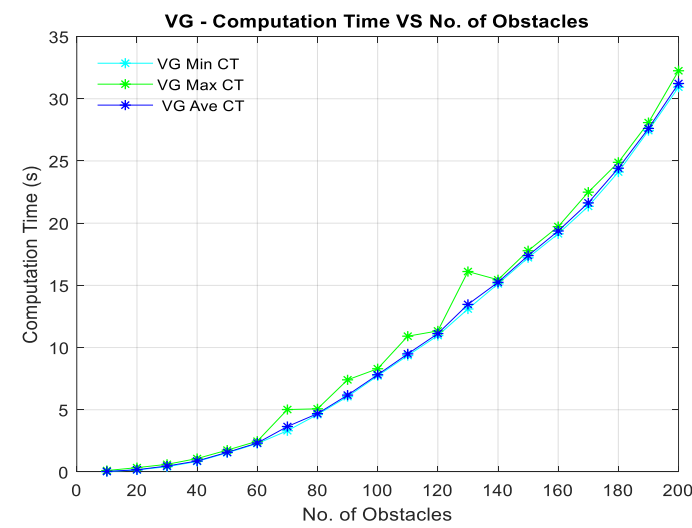

(b)

Figure 6. Computation time with 10 to 200 various obstacles numbers; (a) DAPF, (b) VG

\subsection{The effect of path pruning in DAPF}

Figures 7(a) and (b) show the path lengths produced by VG and DAPF, respectively. It is worth to note that in the simulations, path pruning has not been implemented in DAPF. From the figure, it can be seen that the resulting minimum, average, and maximum path lengths of DAPF are slightly longer than those of VG. From Figure 7(a), it can be seen that the results of minimum, average and maximum path length were identical. In 40 obstacles environments, the resulting path was 1339 units. The graph fluctuates drastically as a result of local minima problems. The robot needed to remove the local minima points and find a new lowest point surrounding it to reach the target point. This process results in a longer path length. Based on Figure 7(b), the path length produced by the VG method increased consistently. VG produced an optimal path length compared to DAPF. Table 2 illustrates the path length by both methods in scenarios with 50, 100, 150 and 200 obstacles. Compared with VG, the path generated by the DAPF method was $83.4 \%$ of the optimum length in the environment with 50 obstacles, $88.3 \%$ in 100 obstacles, $87.6 \%$ in 150 obstacles and $89.5 \%$ in 200 obstacles. 
Table 1. Computation time in environments with 50, 100,150 and 200 obstacles

\begin{tabular}{ccccc}
\hline $\begin{array}{c}\text { Number of } \\
\text { Obstacle }\end{array}$ & Method & \multicolumn{3}{c}{ Computation time (s) } \\
& & Min & Ave & Max \\
\hline 50 & DAPF & 0.32 & 0.34 & 0.39 \\
& VG & 1.54 & 1.58 & 1.76 \\
100 & DAPF & 0.96 & 0.99 & 1.04 \\
& VG & 7.73 & 7.82 & 8.30 \\
150 & DAPF & 2.18 & 2.25 & 2.42 \\
& VG & 17.22 & 17.39 & 17.78 \\
200 & DAPF & 6.05 & 6.15 & 6.43 \\
& VG & 30.93 & 31.23 & 32.25 \\
\hline
\end{tabular}

Table 2. Lengths of the paths produced by DAPF and VG

\begin{tabular}{ccc}
\hline $\begin{array}{c}\text { Number of } \\
\text { Obstacle }\end{array}$ & Method & $\begin{array}{c}\text { Path length } \\
\text { (unit) }\end{array}$ \\
\hline 50 & DAPF & 1214.7 \\
& VG & 1065.2 \\
100 & DAPF & 1195.4 \\
& VG & 1070.5 \\
150 & DAPF & 1209.5 \\
& VG & 1076 \\
200 & DAPF & 1208.4 \\
& VG & 1094 \\
\hline
\end{tabular}

Figure 8 illustrates the path lengths produced by DAPF with path pruning and VG. DAPF with path pruning was found to have a shorter path compared to those without path pruning. From Figure $8(a)$, with 40 obstacles, the produced path length was 1140 units. It proved that DAPF with pruning could generate a shorter path although needed to encounter the local minima problems. Table 3 shows the resulting pruned path length of DAPF. DAPF with path pruning technique successfully generated shorter paths with greater than $96 \%$ of those planned by VG in all scenarios with obstacles number between 50 and 200 . The comparison of path lengths of DAPF with and without pruning is depicted in Figure 9.

From Figure 9, DAPF with pruning yielded shorter paths for the entire scenarios as mentioned in Table 3 compared to DAPF without pruning in Table 2. DAPF with path pruning has been successfully proven to produce a shorter path by an average of $10 \%$ compared to DAPF without path pruning. This is important in saving the robot energy as the robot traverses the path.

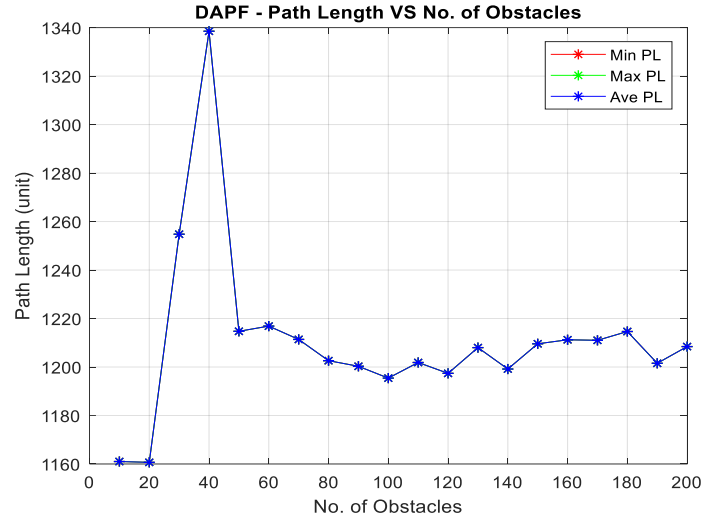

(a)

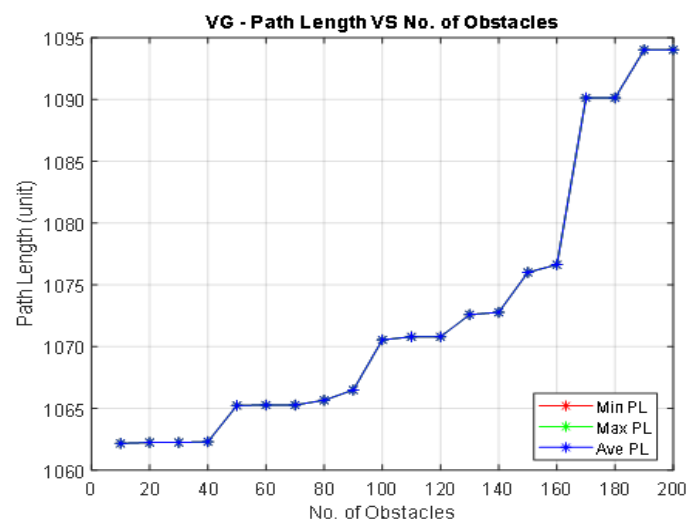

(b)

Figure 7. Path length generated by; (a) DAPF and (b) VG

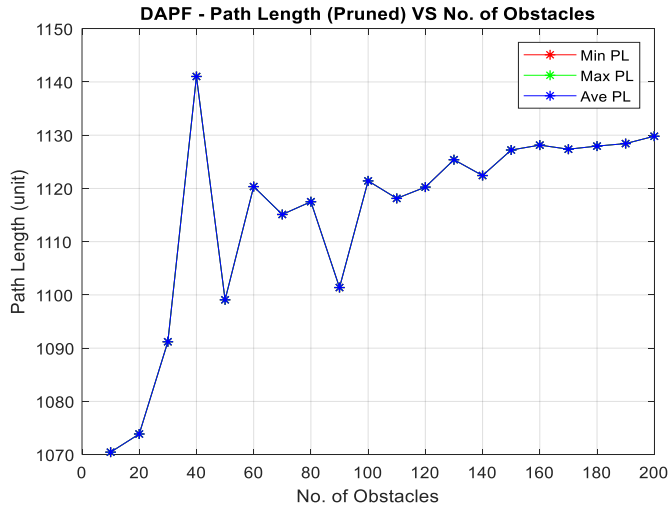

(a)

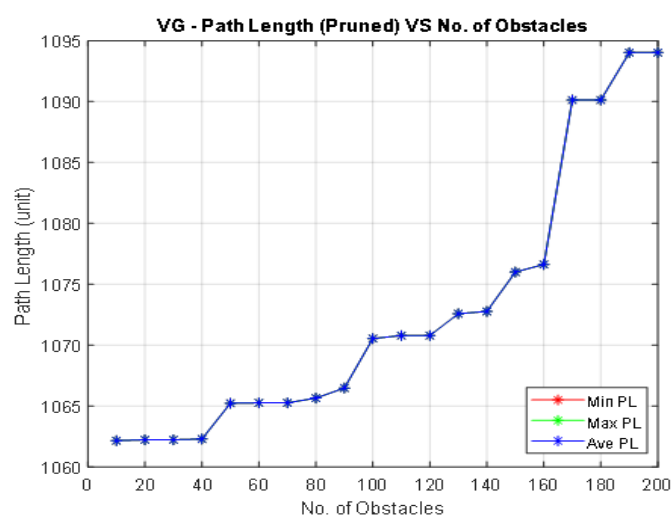

(b)

Figure 8. Path length generated by; (a) DAPF with path pruning and (b) VG 
Table 3. Lengths of planned pruned path for dynamic PF and VG

\begin{tabular}{ccc}
\hline Number of Obstacle & Method & Pruned Path length (unit) \\
\hline 50 & DAPF & 1099.1 \\
& VG & 1065.2 \\
100 & DAPF & 1121.4 \\
& VG & 1070.5 \\
150 & DAPF & 1127.2 \\
& VG & 1076 \\
200 & DAPF & 1129.8 \\
& VG & 1094 \\
\hline
\end{tabular}

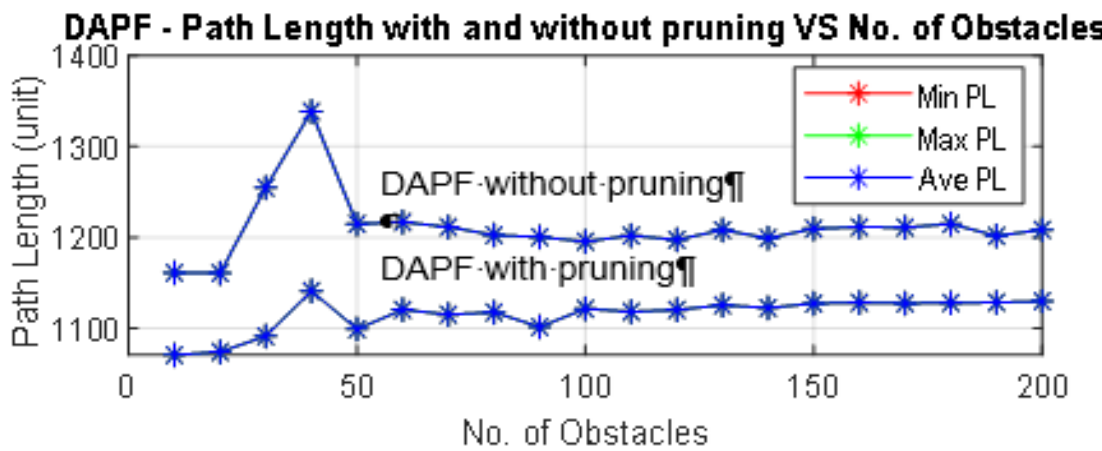

Figure 9. Comparison of DAPF path length with and without path pruning

\section{CONCLUSION}

In this paper, dynamic artificial potential field (DAPF) with path pruning method has been proposed for robot path planning in known environments. The proposed algorithm has successfully overcome the local minima problems of conventional PF. The algorithm is also capable of producing a path with low computation time in obstacle-rich environments, as it is crucial for real-time path planning applications. DAPF has also been validated to produce paths with shorter lengths, which is close to $95 \%$ of those produced by the visibility graph (VG) method. Besides that, DAPF has also been proven to be complete. Therefore, the proposed algorithm fulfills the criterion of path planning.

\section{ACKNOWLEDGEMENTS}

Authors like to give appreciations to Pejabat Penerbitan of Universiti Tun Hussein Onn Malaysia (UTHM) for financial support under student welfare instituition.

\section{REFERENCES}

[1] R. B. Omar, C. K. N. A. H. Che Ku Melor, and E. N. Sabudin, "Performance comparison of path planning methods," ARPN Journal of Engineering and Applied Sciences, vol. 10, no. 19, pp. 8866-8872, 2015.

[2] S. K. Debnath et al., "Flight cost calculation for unmanned air vehicle based on path length and heading angle change," International Journal of Power Electronics and Drive Systems (IJEPDS), vol. 11, no. 1, pp. 382-389, 2020, doi: 10.11591/ijpeds.v11.i1.pp382-389.

[3] Y. Yang, J. Pan, and W. Wan, "Survey of optimal motion planning," IET Cyber-Systems and Robotics, vol. 1, no. 1, pp. 13-19, 2019, doi: 10.1049/iet-csr.2018.0003.

[4] R. bin Omar, "Path Planning For Unmanned Aerial Vehicles Using Visibility Line-Based Methods," Doctor of Philosophy at the University of Leicester, 2011.

[5] X. Chen and X. Chen, "The UAV dynamic path planning algorithm research based on Voronoi diagram," The 26th Chinese Control and Decision Conference (2014 CCDC), Changsha, China, 2014, pp. 1069-1071, doi: 10.1109/CCDC.2014.6852323.

[6] C. Peng, X. Lu, J. Dai, and L. Yin, "Research of path planning method based on the improved Voronoi diagram," 2013 25th Chinese Control and Decision Conference (CCDC), Guiyang, China, 2013, pp. 2940-2944, doi: 10.1109/CCDC.2013.6561448.

[7] M. Kloetzer, C. Mahulea, and R. Gonzalez, "Optimizing cell decomposition path planning for mobile robots using different metrics," 2015 19th International Conference on System Theory, Control and Computing (ICSTCC), Cheile Gradistei, Romania, 2015, pp. 565-570, doi: 10.1109/ICSTCC.2015.7321353. 
[8] D. H. Kim et al., "Path tracking control coverage of a mining robot based on exhaustive path planning with exact cell decomposition," 2014 14th International Conference on Control, Automation and Systems (ICCAS 2014), Gyeonggi-do, Korea (South), 2014, pp. 730-735, doi: 10.1109/ICCAS.2014.6987875.

[9] L. Lulu and A. Elnagar, "A comparative study between visibility-based roadmap path planning algorithms," 2005 IEEE/RSJ International Conference on Intelligent Robots and Systems, Edmonton, AB, Canada, 2005, pp. 3263-3268, doi: 10.1109/IROS.2005.1545545.

[10] T. T. N. Nguyet, T. Van Hoai, and N. A. Thi, "Some advanced techniques in reducing time for path planning based on visibility graph," 2011 Third International Conference on Knowledge and Systems Engineering, Hanoi, Vietnam, 2011, pp. 190-194, doi: 10.1109/KSE.2011.37.

[11] S. K. Debnath et al., "A review on graph search algorithms for optimal energy efficient path planning for an unmanned air vehicle," Indonesian Journal of Electrical Engineering and Computer Science (IJEECS), vol. 15, no. 2, pp. 743-750, 2019, doi: 10.11591/ijeecs.v15.i2.pp743-749.

[12] S. K. Debnath, R. Omar, N. Badariyah, and N. B. Binti Abdul Latip, "Comparison of different configuration space representations for path planning under combinatorial method," Indonesian Journal of Electrical Engineering and Computer Science (IJEECS), vol. 14, no. 3, pp. 401-408, 2019, doi: 10.11591/ijeecs.v10.i1.pp401-408.

[13] G. Y. Jeon and J. W. Jung, "Water sink model for robot motion planning," Sensors, vol. 19, no. 6, 2019, Art. no. 1269 , doi: $10.3390 / \mathrm{s} 19061269$.

[14] N. Tran, D. T. Nguyen, D. L. Vu, and N. V. Truong, "Global path planning for autonomous robots using modified visibility-graph," 2013 International Conference on Control, Automation and Information Sciences (ICCAIS), Nha Trang, Vietnam, 2013, pp. 317-321, doi: 10.1109/ICCAIS.2013.6720575.

[15] Y. Ma, G. Zheng, and W. Perruquetti, "Cooperative path planning for mobile robots based on visibility graph," Proceedings of the 32nd Chinese Control Conference, Xi'an, China, 2013, pp. 4915-4920

[16] N. B. A. Latip, R. Omar, and S. K. Debnath, "Optimal path planning using equilateral spaces oriented visibility graph method," International Journal of Electrical and Computer Engineering (IJECE), vol. 7, no. 6, pp. 3046-3051, 2017, doi: 10.11591/ijece.v7i6.pp3046-3051.

[17] N. B. Binti Abdul Latip, "Computationally Efficient Path Planning Algorithms In Obstacle-Rich Environments Based On Visibility Graph Method,” Thesis, Universiti Tun Hussein Onn Malaysia, 2018.

[18] Y. Koren, S. Member, J. Borenstein, and A. Arbor, "Potential Field Methods and Their Inherent Limitations for Mobile Robot Navigation," Proceedings. 1991 IEEE International Conference on Robotics and Automation, Sacramento, CA, USA, 1991, pp. 1398-1404, vol. 2, doi: 10.1109/ROBOT.1991.131810.

[19] Y. Cen, L. Wang, and H. Zhang, "Real-time obstacle avoidance strategy for mobile robot based on improved coordinating potential field with genetic algorithm," 2007 IEEE International Conference on Control Applications, Singapore, 2007, pp. 415-419, doi: 10.1109/CCA.2007.4389266.

[20] F. A. L. Lifen, B. S. Ruoxin, C. L. Shuandao, and D. W. Jiang, "Path planning for UAVS based on improved artificial potential field method through changing the repulsive potential function," 2016 IEEE Chinese Guidance, Navigation and Control Conference (CGNCC), Nanjing, China, 2016, pp. 2011-2015, doi: 10.1109/CGNCC.2016.7829099.

[21] Y. Liu and Y. Zhao, "A virtual-waypoint based artificial potential field method for UAV path planning," 2016 IEEE Chinese Guidance, Navigation and Control Conference (CGNCC), Nanjing, China, 2016, pp. 949-953, doi: 10.1109/CGNCC.2016.7828913.

[22] E. N. Sabudin, R. Omar, and C. K. A. N. H. Che Ku Melor, "Potential field methods and their inherent approaches for path planning," ARPN Journal of Engineering and Applied Sciences, vol. 11, no. 18, pp. 10801-10805, 2016.

[23] O. Khatib, "Real-time obstacle avoidance for manipulators and mobile robots," Proceedings. 1985 IEEE International Conference on Robotics and Automation, St. Louis, MO, USA, 1985, pp. 500-505, doi: 10.1109/ROBOT.1985.1087247.

[24] M. Wang, Z. Su, D. Tu, and X. Lu, "A Hybrid Algorithm Based on Artificial Potential Field and BUG for Path Planning of Mobile Robot," Proceedings of 2013 2nd International Conference on Measurement, Information and Control, Harbin, China, 2013, pp. 1393-1398, doi: 10.1109/MIC.2013.6758219.

[25] S. Wang and H. Min, "Experience mixed the modified artificial potential field method," 2013 IEEE/RSJ International Conference on Intelligent Robots and Systems, Tokyo, Japan, 2013, pp. 4823-4828, doi: 10.1109/IROS.2013.6697052.

[26] H. A. Guanghui Li, Y. Tamura, A. Yamashita, "Effective improved artificial potential field-based regression search method for autonomous mobile robot path planning," International Journal of Mechatronics and Automation, vol. 3, no. 3, pp. 141-170, 2013, doi: 10.1504/IJMA.2013.055612.

[27] J. Sfeir, M. Saad, and H. Saliah-Hassane, "An improved Artificial Potential Field approach to real-time mobile robot path planning in an unknown environment," 2011 IEEE International Symposium on Robotic and Sensors Environments (ROSE), Montreal, QC, Canada, 2011, pp. 208-213, doi: 10.1109/ROSE.2011.6058518.

[28] J.-W. Park, H.-J. Kwak, Y.-C. Kang, and D. W. Kim, "Advanced Fuzzy Potential Field Method for Mobile Robot Obstacle Avoidance," Computational Intelligence and Neuroscience, vol. 2016, 2016, Art. no. 6047906, doi: 10.1155/2016/6047906.

[29] G. Li et al., "An Improved Artificial Potential Field-based SImultaneous FORward Search (Improved APF-based SIFORS) method for robot path planning," 2015 12th International Conference on Ubiquitous Robots and Ambient Intelligence (URAI), Goyangi, Korea (South), 2015, pp. 330-335, doi: 10.1109/URAI.2015.7358965.

[30] S. K. Debnath, R. Omar, and N. B. A. Latip, "A review on energy efficient path planning algorithms for unmanned air vehicles,” Computational Science and Technology, vol. 481, pp. 523-532, 2019, doi: 10.1007/978-981-13-2622-6_51. 


\section{BIOGRAPHIES OF AUTHORS}

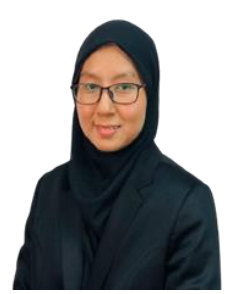

Elia Nadira Sabudin received her first degree in electrical engineering from Universiti Tun Hussein Onn Malaysia (UTHM) in 2008. She received her Master of electical and electronic engineering from Universiti Tun Hussein Onn Malaysia (UTHM) in 2012. She is currently a $\mathrm{PhD}$ student in Faculty of Electrical \& Electronic Engineering in the Universiti Tun Hussein Onn Malaysia (UTHM). Her research interests in UAV path planning approach based on Potential field method.

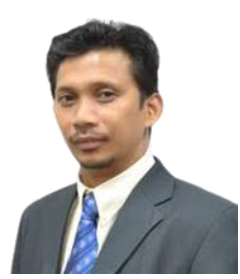

Rosli Omar is currently an associate professor at Faculty of Electrical and Electronic Engineering, Universiti Tun Hussein Onn Malaysia (UTHM). He received his $\mathrm{PhD}$ in engineering from University of Leicester, United Kingdom in 2012. His first degree was accomplished in 1999 in instrumentation and control engineering. He then pursued his master's degree in electrical engineering, specialised in system identification and graduated in 2002. Both his first dan master's degrees are from Universiti Teknologi Malaysia (UTM). His research interests are in robotic engineering, autonomous system and system identification.

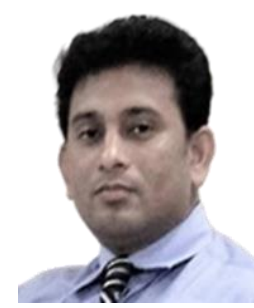

Sanjoy Kumar Debnath is PhD student of Faculty of Electrical \& Electronic Engineering from Universiti Tun Hussein Onn Malaysia (UTHM). He completed his bachelor' degree of Engineering from the Presidency University Bangladesh in 2008 and his Master of engineering from Universiti Teknologi Malaysia in 2014. He joined a research on "Optimal Energy Efficient Path Planning for an Unmanned Air Vehicle (UAV) in Obstacle-Rich Environment" in 2015 at UTHM.

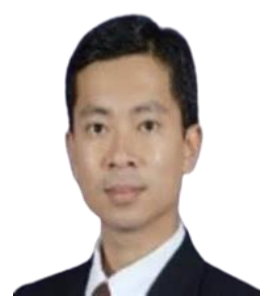

Muhammad Suhaimi Sulong is a Senior Lecturer and Head of Technology Programme at Faculty of Technical and Vocational Education, Universiti Tun Hussein Onn Malaysia (UTHM). $\mathrm{He}$ is a joint founder of Microelectronics and Nanotechnology-Shamsuddin Research Centre (MiNT-SRC) UTHM established in November 2006 and joining a focus group research in IoT Technology Unit. He had experienced joining the Nano Research Group in the School of Electronics and Computer Science, University of Southampton, UK (2003-2004) and also as Visiting Scholar at University of South Australia (2011-2012). He received his Doctorate in Higher Education from Universiti Selangor (UNISEL), Malaysia in 2017, M.Sc. Microelectronics from Universiti Kebangsaan Malaysia (UKM) in 2005, and B.Eng. (Hons) Electrical Engineering (Computer Engineering) from UTHM in 2002. His current research interests include IC design, IoT-based research, training and skill, curriculum development, internationalization of higher education, and e-learning. 